\title{
Only, at least, more, and less
}

Thomas Brochhagen and Elizabeth Coppock

Claim. We argue that the superlative modifiers at least and at most (i) depend on the strength ranking over the answers to the question under discussion (QUD) for their interpretation and (ii) are inquisitive, unlike more and less/fewer. We make both of these claims precise with a synthesis of Beaver and Clark's (2008) pragmatic scales with unrestricted inquisitive semantics (Ciardelli et al. 2009). Our theory captures the following facts.

Truth conditions. The variants of the following sentence are true under the same circumstances.

(1) John scored \{at least five, more than four goals.

Focus sensitivity. At least is focus-sensitive, as Krifka (1999) points out. Our example:

(2) a. John should at least invite the [postdoc $]_{F}$ to lunch.

b. John should at least invite the postdoc [to lunch $]_{F}$.

(2a) implies that John should invite someone to lunch, while (2b) does not; in (2b) dinner would also be fine. (2b) implies that John should invite the postdoc to something, whereas (2a) does not.

Relationship to only. As discussed by Coppock and Beaver (2011), scalar exclusives like only, just and merely presuppose at least, and assert at most.

Authoritative readings. As Büring (2008) points out, (3) has both an 'authoritative reading', on which it would be used to inform the interlocutor what the acceptable page lengths are, and a 'speaker insecurity' reading, on which the speaker does not know what the required length of the paper is, but believes it to be over 10 pages.

(3) The paper has to be at least 10 pages long.

Scalar implicatures. It is usually assumed that three conversationally implicates 'not four' because four is a stronger alternative that the speaker would have chosen had it been appropriate. If that is the case, then, as Krifka (1999) points out, at least three should conversationally implicate "not at least four' as well by the same logic. But it does not.

(4) Bertha drank (at least) three beers.

Ignorance implicature. Superlative modifiers typically give rise to an ignorance implicature while comparative modifiers do not (Geurts and Nouwen 2007).

(5) A hexagon has \{at least, more than 5 sides.

(Nouwen 2010)

Initial Hypothesis: A QUD-based analysis of superlative modifiers.

Let $s$ be a discourse context, let $\hat{s}$ be the QUD in this discourse context, and let $\geq_{s}$ be the pragmatic strength ranking over the answers to the question under discussion. Questions are sets of propositions, i.e. sets of possible worlds. 
For example, suppose the question in $s$ is 'Who snores?', and $w_{10}$ is the world where Ann snores and Bill does not snore, $w_{11}$ is the world where they both snore, and so on. Then $\hat{s}=\{a, b, a \& b\}$ where $a=\left\{w_{10}, w_{11}\right\}$ 'Ann snores', $b=\left\{w_{01}, w_{11}\right\}$ 'Bill snores', and $a \& b=\left\{w_{11}\right\}$ 'Ann and Bill snore'. If $a \& b$ is stronger than both $a$ and $b$, and $a$ and $b$ are unranked with respect to each other then $\geq_{s}=\{\langle a \& b, a\rangle,\langle a \& b, b\rangle,\langle a, a\rangle,\langle b, b\rangle,\langle a \& b, a \& b\rangle\}$.

Our proposal for at least and at most builds on the following (Coppock and Beaver 2011).

$$
\begin{aligned}
& \text { a. } \quad \operatorname{MIN}_{s}(p)=\lambda w \cdot \exists p^{\prime} \in \hat{s}: p^{\prime}(w) \wedge p^{\prime} \geq_{s} p \\
& \text { b. } \quad \operatorname{MAX}_{s}(p)=\lambda w \cdot \forall p^{\prime} \in \hat{s}: p^{\prime}(w) \rightarrow p \geq_{s} p^{\prime}
\end{aligned}
$$

$\operatorname{MIN}(p)$ says that there is a true answer to the QUD that is at least as strong as $p$, and $\operatorname{MAX}(p)$ says that all answers to the QUD that are stronger than $p$ are false. These are the presupposed and asserted meaning components that Beaver and Clark (2008) assign to only.

Our initial hypothesis is that, in state $s$, at least $p$ means $\operatorname{MIN}_{s}(p)$, and at most $p$ means $\operatorname{MAX}_{s}(p)$. This analysis accounts for the relationship between superlatives and exclusives, the truth conditions of superlative modifiers, their focus-sensitivity, their ability to combine with entailment and non-entailment scales, and their ambiguity under modals. (See Coppock and Brochhagen 2012 for more detail.) But the facts related to implicatures remain mysterious.

At least in inquisitive semantics. Our initial hypothesis does not explain the contrast between superlative and comparative modifiers with respect to ignorance implicatures. It is basically equivalent to Büring's analysis, and although Büring purports to explain this contrast, his explanation is conceptually problematic. According to Büring, at least "amounts to" a disjunction between the prejacent and its higher-ranked alternatives. This means, according to Büring, that it is subject to the following implicature schema:

(7) If a speaker utters $p$ or $q$, it is implied that (i) in all of the speaker's doxastic alternatives $p \vee q$ and (ii-a) in some of the speaker's doxastic alternatives $p$, and (ii-b) in some $q$.Büring (2008: 114f)

But the only level at which the meaning of at least is a disjunction in Büring's theory is in the meta-language. We cannot make our theories of implicature depend on the syntactic form of the meta-language description of the meaning of a sentence.

If we turn to inquisitive semantics, we can give substance to the notion that at least is like a disjunction. In inquisitive semantics, declarative sentences and interrogative sentences both denote sets of sets of possible worlds. For example, while ' $a$ or $b$ ' classically denotes the set of worlds where either $a$ or $b$ is true, ' $a$ or $b$ ' denotes a set containing two sets of worlds in inquisitive semantics: those where $a$ is true, and those where $b$ is true.

We propose that at least $p$ denotes the set containing all possibilities $p^{\prime}$ such that $p^{\prime}$ is at least as strong as $p$ according to the pragmatic strength ranking over answers to the QUD. For example (cf. Figure 1):

$$
\llbracket \text { At least Ann snores } \rrbracket^{s}=\left\{p \mid p \geq_{s} a\right\}=\{a, a b\}=\left\{\left\{w_{10}, w_{11}\right\},\left\{w_{11}\right\}\right\}
$$

As Figure 1 illustrates, At least Ann snores and Ann snores have the same informational content. But the at least sentence is interactive (i.e. contains multiple possibilities). 

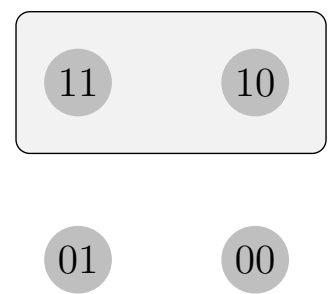

(a) Ann snores

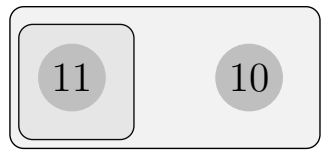

01

00

(b) At least Ann snores

Figure 1: Attentive and non-attentive propositions

Ignorance implicatures. The reason that At least Ann snores conveys an ignorance implicature is that it draws attention to the issue of whether Ann or anyone else snores by uttering this sentence, and it doesn't make sense to draw attention to an issue if you already know how to settle it. This intuition can be formalized with the notion of interactive sincerity. ${ }^{1}$

\section{(9) Maxim of Interactive Sincerity}

If $\phi$ is interactive, then $\phi$ is interactive in the speaker's information state.

A proposition is interactive if it contains multiple possibilities; a proposition is interactive in a given information state, if it is still interactive once all of the worlds that are in that information state are removed from it.

Suppose that the speaker knows that Ann snores and nobody else does (hence Bill does not). Then the speaker's information state is $\left\{w_{10}\right\}$, the set of worlds where that is true (a singleton set in this tiny example). The denotation of At least Ann snores restricted to that state gives a proposition consisting of the state itself. This proposition is not interactive, so Interactive Sincerity is violated. Only when the speaker's information set is consistent with higher-ranked alternatives can an at least sentence satisfy Interactive Sincerity. Hence the ignorance implicature.

Scalar and exhaustivity implicatures. In response to the question 'Who came to the party?', the answer 'Anna' implies that nobody else came to the party whereas 'at least Anna' does not imply that. We can refer to this as the 'exhaustivity implicature'.

As we showed above, the two responses have the same informative content, but one is interactive and the other is not. This difference can be exploited to explain the contrast with respect to exhaustivity. Following Balogh (2009), we assume that the exhaustification of a proposition $P$ in the context of a question under discussion $Q$ (also an inquisitive-style proposition) is the result of removing worlds $w$ from each of the possibilities $p$ in $P$ such that $w$ is contained in one of the possibilities in $Q$ that is not implied by $p$. We formalize this as follows:

(10) Exhaustification

$$
\operatorname{EXH}(P, Q)=\left\{p-q \mid p \in P \& q=\left\{w \mid \exists q^{\prime} \in Q\left[w \in q^{\prime} \& p \nsubseteq q^{\prime}\right]\right\}\right\}
$$

In our example, the question is 'Who snores?', and the possible answers are: 'Ann', 'Bill', and 'Ann and Bill'. Applied to 'Ann snores' in the context of the question 'Who snores?', this yields the result that nobody else snores. From the single possibility in the denotation of 'Ann snores', namely

\footnotetext{
${ }^{1}$ This is a generalization of the Maxim of Inquisitive Sincerity as defined by Pruitt and Roelofsen (2011), which has inquisitive where we have interactive.
} 


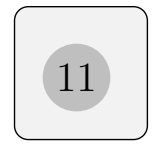

01

\section{0}

00

(a) $\operatorname{EXH}(' A n n ')$

(b) $\operatorname{EXH('at~least~Ann')~}$

Figure 2: Exhaustification of two propositions with respect to 'Who snores?'

$\left\{w_{10}, w_{11}\right\}$, we eliminate the world $w_{11}$, because that world is part of a non-implied alternative, 'Bill snores'. The result is $\left\{\left\{w_{10}\right\}\right\}$, as shown in Figure 2(a).

For 'At least Ann snores', we start with possibilities, $a=\left\{w_{10}, w_{11}\right\}$ and $a \& b=\left\{w_{11}\right\}$. Exhaustification causes $w_{11}$ to be removed from the first one. But nothing is removed from the second one, because every alternative is either disjoint from it or implied by it, since it contains only one world. The result is $\left\{\left\{w_{10}\right\},\left\{w_{11}\right\}\right\}$, as depicted in Figure 2(b). Hence exhaustification does not result in the inference that Bill does not snore in this case.

Summary. We argue that at least and at most denote MIN and MAX respectively, and that they, unlike comparative modifiers, are inquisitive. Together, these assumptions explain all of the data described at the beginning. Beyond providing what we argue to be the most empirically successful analysis of at least on the market (see Coppock and Brochhagen 2012 for more detail), we have also brought pragmatic scales into inquisitive semantics. It will be interesting to see what other interactions we may find between pragmatic strength and inquisitivity in future research.

\section{References}

Balogh, K. (2009). Theme with Variations: A Context-based Analysis of Focus. PhD thesis, University of Amsterdam.

Beaver, D. I. and Clark, B. Z. (2008). Sense and Sensitivity: How Focus Determines Meaning. Wiley-Blackwell, Chichester.

Büring, D. (2008). The Least at least Can Do. In Chang, C. B. and Haynie, H. J., editors, Proceedings of the 26th West Coast Conference on Formal Linguistics, pages 114-130. Cascadilla Press, Somerville, MA.

Ciardelli, I., Groenendijk, J., and Roelofsen, F. (2009). Attention! Might in inquisitive semantics. In Ito, S. and Cormany, E., editors, Proceedings from Semantics And Linguistic Theory XIX. CLC Publications.

Cohen, A. and Krifka, M. (2011). Superlative quantifiers as modifiers of meta-speech acts. In Partee, B. H., Glanzberg, M., and Skilters, J., editors, The Baltic International Yearbook of Cognition, Logic and Communication. New Prairie Press.

Coppock, E. and Beaver, D. (2011). Sole sisters. In Ashton, N., Chereches, A., and Lutz, D., editors, Proceedings of Semantics and Linguistic Theory (SALT) 21, pages 197-217. Rutgers University, eLanguage.

Coppock, E. and Brochhagen, T. (2012). Raising and resolving issues with scalar modifiers. Submitted to Semantics \& Pragmatics.

Geurts, B. and Nouwen, R. (2007). At least et al.: The semantics of scalar modifiers. Language, 83:533-559.

Krifka, M. (1999). At least some determiners aren't determiners. In Turner, K., editor, The Semantics/Pragmatics Interface from Different Points of View, pages 257-291. Elsevier, Oxford.

Nouwen, R. (2010). Two kinds of modified numerals. Semantics \& Pragmatics, 3:1-41.

Pruitt, K. and Roelofsen, F. (2011). Disjunctive questions: prosody, syntax, and semantics. Presented at a seminar at the Georg August Universitä̈ Göttingen, April 2011. 